\title{
PTP1B Inhibitors as Potential Target for Type II Diabetes
}

\author{
Tanvi A Deshpande, Martina Isshak and Ronny Priefer* \\ Massachusetts College of Pharmacy and Health Sciences University, Boston, USA
}

Submission: November 11, 2020; Published: December 07, 2020

*Corresponding author: Ronny Priefer, Massachusetts College of Pharmacy and Health Sciences University, 179 Longwood Avenue, Boston, MA, USA

\begin{abstract}
Diabetes mellitus is a metabolic disorder characterized by elevated blood sugar levels resulting primarily from either a lack of insulin production or insulin resistance. Insulin elicits its effects by activating the Insulin Receptor (IR) which is responsible for glucose uptake and promotes insulin signaling. Protein tyrosine phosphatase 1B (PTP1B) is a negative regulator of IR signaling and is responsible for dephosphorylating the tyrosine phosphorylated IR. Inhibition of PTP1B could thus promote glucose uptake [1]. Early PTP1B inhibitors, such as charged active site-directed analogs, showed poor cell membrane permeability. Subsequent uncharged mimetics overcome this initial hurdle however specificity over T-cell protein tyrosine phosphatase (TCPTP) was another challenge that needed to be overcome. Herein is an overview of the challenges faced for targeting PTP1B. These include the evaluation of compounds that target the active site, allosteric site, and finally covalent inhibition of this potentially viable drug target.
\end{abstract}

Keywords: PTP1B; Type 2 diabetes mellitus; TCPTP; Non-selective inhibitors; Selective inhibitors; Covalent inhibitors

\section{Introduction}

Diabetes mellitus is a metabolic disorder characterized by elevated blood sugar levels resulting primarily from either a lack of insulin production or insulin resistance. Based on its pathophysiology, the disease is categorized as either Type 1, Type 2, or gestational diabetes. Type 2 diabetes accounts for nearly $90 \%$ of the total diabetes cases. The International Diabetes Federation reported that globally, approximately 415 million people were afflicted with diabetes in 2015 and this number is expected to rise to 642 million by 2040 [2]. According to the World Health Organization, diabetes is the 7 th leading cause of death globally [2].

Type 1 diabetes (T1D) is a consequence of the autoimmune destruction of insulin producing beta cells in the pancreas. This leads to lack of insulin within the bloodstream, resulting in hyperglycemia. Between 5 to $10 \%$ of patients with diabetes are diagnosed with this form [3]. Due to body's inability to produce insulin, the treatment involves administration of this vital hormone. Conversely, non-insulin dependent or Type 2 diabetes (T2D) is characterized by insulin resistance in peripheral tissues. In this case the resultant decrease in cellular glucose uptake combined with decreased insulin secretion results in hyperglycemia. This can lead to several secondary complications such as atherosclerosis, microangiopathy, renal dysfunction and failure, cardiac abnormalities, diabetic retinopathy, and ocular disorders [4]. Approximately 90 to $95 \%$ of diabetic patients have T2D [3]. There are several classes of antidiabetic drugs available on the market for the treatment of T2D. Sulfonylureas and meglitinides stimulate the pancreas to release more insulin [5], biguanides decrease glucose production4, thiazolidinediones increase insulin sensitivity [6], $\alpha$-glucosidase inhibitors slow down the absorption of carbohydrates ingested [7], dipeptidylpeptidase-4 inhibitors intensify the effect of certain intestinal hormones involved in the control of blood sugar levels, 4 sodiumglucose transporter inhibitors help eliminate glucose in the urine, 4 and glucagon-like peptide 1 agonists mimic the effects of certain intestinal hormones involved in the control of blood sugar levels [8]. In addition, non-pharmacological treatment options include proper diet, exercise, and a general decrease in glucose intake. Gestational diabetes is a condition which can occur during pregnancy. Pregnant patients develop an elevation in their blood glucose which causes other complications. Pregnant women with gestational diabetes are at a higher risk of developing preeclampsia. Gestational diabetes can be prevented through health lifestyle managements such as diet and exercise, however in some cases it may require the use of medications [9]. After delivery, the 
woman's blood sugar usually normalizes, however in some cases it has been shown that they can be predisposed to developing T2D1.

Insulin elicits its effects by activating the Insulin Receptor (IR), a transmembrane Protein Tyrosine Kinase (PTK) that phosphorylates itself as well as its target substrates such as IR substrate (IRS) 1 and 2.8 Upon insulin binding, the insulin receptor undergoes transphosphorylation at various sites. This causes activation of IR, recruitment, and phosphorylation of its substrates (e.g. IRS 1) resulting in downstream signaling [1]. Activated IR is responsible for glucose uptake and promotes insulin signaling whereby insulin acts on the liver and brain to turn off hepatic glucose production, promote glycogen synthesis and lipogenesis on muscles, as well as increase the uptake of glucose for storage as glycogen and triglycerides [1]. Protein tyrosine phosphatase $1 \mathrm{~B}$ is a negative regulator of IR signaling and is responsible for dephosphorylating the tyrosine phosphorylated IR and its substrate proteins. PTPs consist of a large family of proteins encoded by nearly 100 genes [10]. Classical PTPs show some common structural features, one of which is a general acid/base loop (WPD loop). PTP1B is the major insulin receptor phosphatase in muscle and liver1 and like other classical phosphatases also possess the WPD loop spanning from residue Tyr 179 to Pro 185 [11]. In addition, it consists of substrate binding region referred to as the P-loop which possesses the nucleophilic cysteine 215 amino acid which is involved in the hydrolysis of the phosphate ester, a lysine loop ranging from residues 150 to 153, and a tyrosine phosphate (TyrP) recognition loop from Tyr 46 to Val 49 [11] Additionally, the catalytic domain of PTP1B can be divided in to 4 sites- A, B, C, and D site. The A site known as the catalytic pocket is where the pTyr residue of the IR undergoes dephosphorylation. The B-site is a site containing a second pTyr binding region. The pTyr residue here is primarily involved in interactions with Arg 24 and Arg 254. The C- site is a highly solvent exposed site, whereas the D-site is a solvent shielded. It is a small, narrow pocket lined with polar residues such as Lys 120, Lys 116, Asp 181, Ser 216 [12,13]. Recently, a second allosteric pocket has also been identified within PTP1B [14] (Figure 1).

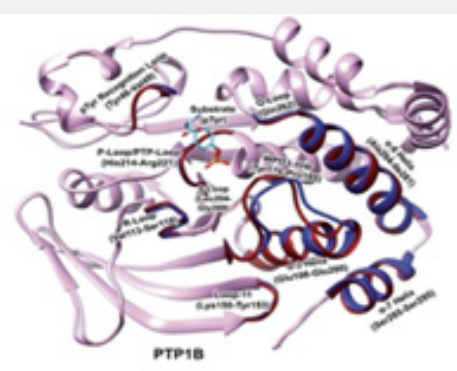

(A)

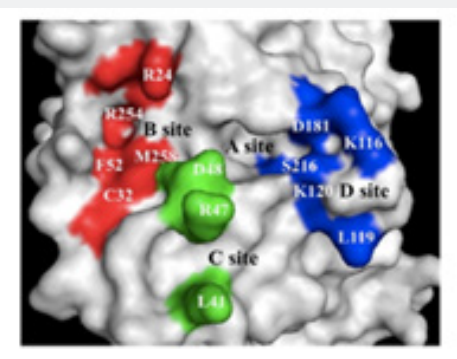

(B)
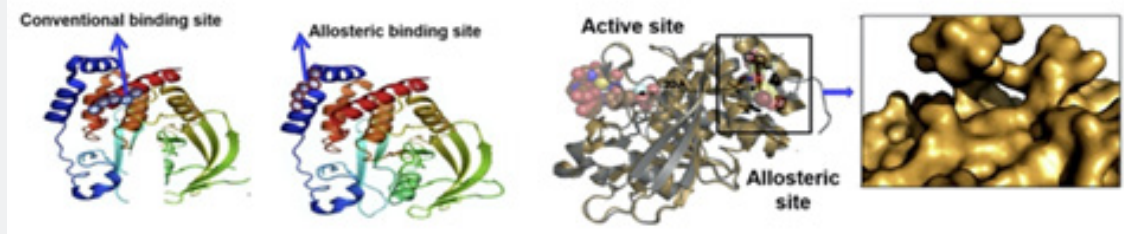

Figure 1: Different loops (A) [image taken from reference 11] and sites (B) [image taken from reference 27] of enzyme PTP1B. Conventional substrate binding site (active site) and the recently identified allosteric binding site [image taken from reference 14].

The active site cysteine has an unusually low pKa value of approximately 5 [15]. The thiolate anion is responsible for attacking the phosphate ester moiety of the substrate. The result is the formation of a phosphorylated enzyme with the subsequent release of the dephosphorylated tyrosine containing peptide [16]. The $2^{\text {nd }}$ steps involves the regeneration of the enzyme by attack of water on the phosphorylated enzyme yielding the dephosphorylated enzyme and the release of inorganic phosphate (Figure 2) [16].

This PTP1B mediated dephosphorylation of the IR terminates insulin action and may ultimately results in insulin resistance. It has been demonstrated that in T2D there is an overexpression PTP1B. It has been demonstrated that the inhibition or deletion of PTP1B leads to an increase in the auto-phosphorylation of the insulin receptor which thus enhances insulin sensitivity and glucose uptake [17]. Mice lacking the expression of PTP1B, due its inhibition or deletion, displayed enhanced insulin sensitivity, prolonged insulin-stimulated phosphorylation of the insulin receptor, resistant to weight gain, and remained insulin sensitive when they were administered a high-fat diet [18]. As a result, inhibition of PTP1B could mimic several actions of insulin such as promotion of glucose uptake as well as inhibition of lipolysis which makes it a promising target in the treatment of T2D [1]. 


\section{Current Research in Diabetes \& Obesity Journal}

Thus the design of PTP1B inhibitors has become an active area of research. Early PTP1B inhibitors developed were active site directed analogs that structurally resembled its substrate.

\section{Non-Selective PTP1B Inhibitors}

Vanadium containing compounds are an example of nonselective p-Tyr mimetics. Vanadium can exist in oxidation states ranging from + II to $+\mathrm{V}$. In aqueous solutions it mainly exists as IV or V [19]. The orthovanadate ion structurally resembles the phosphate group and binds to the phosphate binding site of PTP1B. Peroxidovanadium compounds are able to oxidize the critical active site cysteine residue and irreversibly inhibits the enzyme. Vanadium is also found to inhibit PTPs via an indirect mechanism referred to as a Fenton-like reaction. Interconversion between the vanadyl (IV) and vanadate (V) redox states results in the formation of reactive oxygen species (ROS). These ROS can oxidize cysteine residues of the PTPs including the catalytic cysteine. Some vanadium-based compounds showed promising in vitro results with $\mathrm{IC}_{50}$ in the low $\mu \mathrm{M}$ range (Figure 3 ) however randomized clinical trials for the treatment of diabetes concluded that these drugs caused GI distress, showed low efficacy among diabetic patients, and showed large patient to patient variability [19].

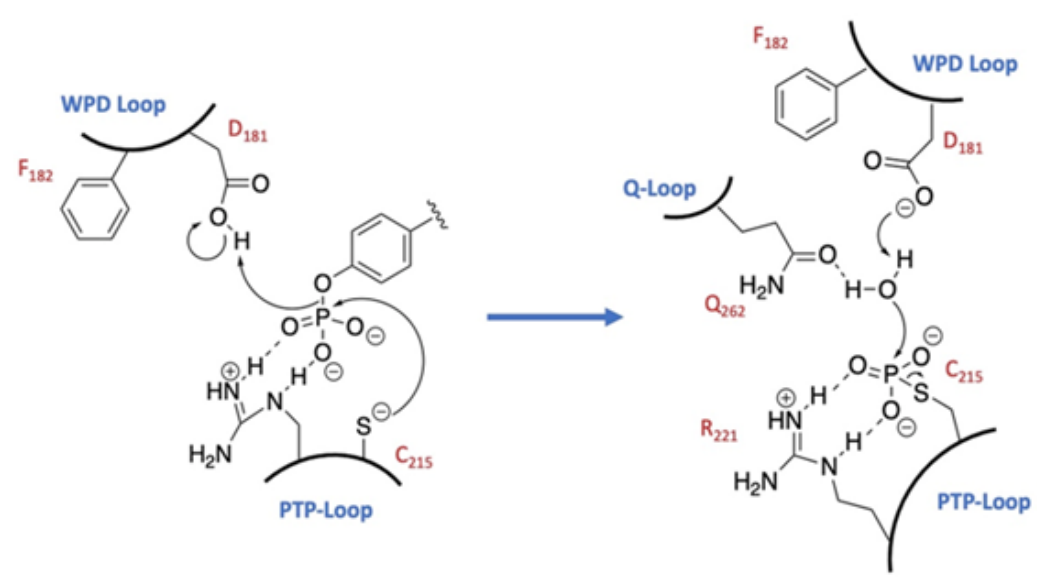

Figure 2: The catalytic mechanism of PTP1B.

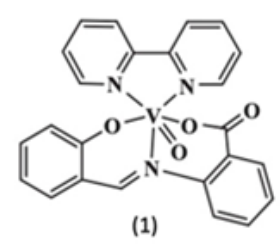

(1)

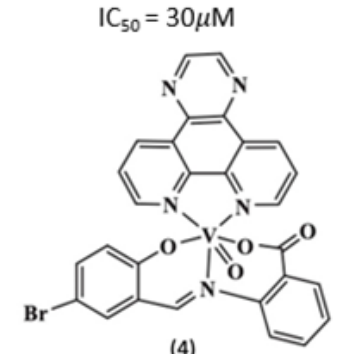

(4)

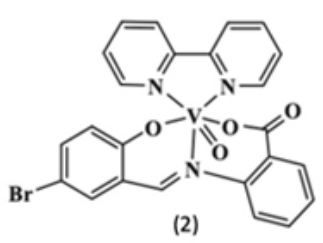

$\mathrm{IC}_{50}=41 \mu \mathrm{M}$<smiles>COP(=O)(O)N1C(=O)c2ccccc2OP1(=O)OCc1cc(Br)ccc1O</smiles>

(5)

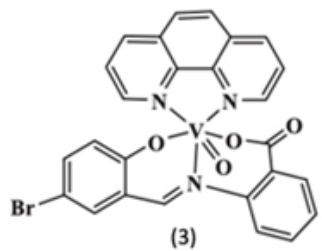

$I_{50}=42 \mu \mathrm{M}$

$\mathrm{IC}_{50}=0.21 \mu \mathrm{M}$

(6)<smiles>COP1(=O)OC(=O)c2ccccc2N1C=C1c2ccc([N+](=O)[O-])cc2OP1(=O)O</smiles>

$I_{50}=44 \mu \mathrm{M}$

Figure 3: Structures of vanadium complexes with known PTP inhibitory activity [19].

Other pTyr mimetics include diflurophosphates (DFMP). With DFMP's, the phosphate portion forms an electrostatic interaction with the pTyr binding pocket whereas the adjacent carbonyl possesses additional H-bonding interactions. Among these series compound 7 (Figure 4) which is a bis-DFMP, emerged as the most potent inhibitor with IC50 and Ki values in sub micromolar levels [4]. However, the dianionic nature of the bis-DMPF scaffold was a limiting factor in terms of cell membrane permeability. 


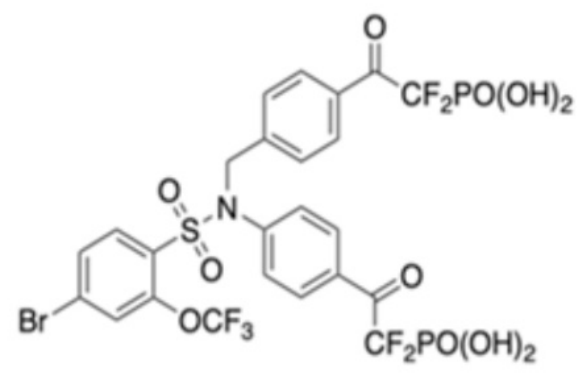

(7)

$$
\mathrm{IC}_{50}=0.5 \mu \mathrm{M}
$$

Figure 4: Most potent (in-vitro) DFMP containing PTP1B inhibitor [4].

Other possible pTyr mimetics, suggested by structure-based drug design, was the dicarboxylic acid containing malonate group. 4 These compounds were regarded as the most successful non-phosphorous containing pTyr mimetics. They include
O-malonyltyrosine (OMT) (8) [20], fluoro-0-malonyltyrosine (FOMT) (9) [21] and 3-carboxy-0-carboxymethyl tyrosine (10) [4] analogs (Figure 5).<smiles>[R]c1ccc(OC(C(=O)O)C(=O)O)cc1</smiles>

(8)<smiles>[R]c1ccc(OC(C(=O)O)(C(=O)O)C(=O)O)cc1</smiles>

(9)<smiles>[R]c1ccc(OCC(=O)O)c(C(=O)O)c1</smiles>

(10)

Figure 5: Scaffolds O-malonyltyrosine (OMT) (8), fluoro-O-malonyltyrosine (FOMT) (9), and 3-carboxy-O-carboxymethyl tyrosine (10) [4].

Another class of active site directed PTP1B inhibitors are the benzofuran/benzothiophene biphenyl derivatives4. Ertiprotafib (Figure 6), a mono carboxylic acid benzothiophene pTyr mimetic was developed at Wyeth as a potential PTP1B inhibitor and reached clinical trials [4]. However, it has multiple mechanisms of actions, such as PTP1B inhibition, dual PPAAR $\alpha /$ PPAR $\gamma$ agonism, and IKK- $\beta$ inhibition [22]. Side effects resulting from these varied MOA was a major reason why it was discontinued in the Phase II trials [22]. Additionally, Novo Nordisk identified the 2-(oxalylamino)-benzoic acid (OBA) scaffold based on scintillation proximity technology high throughput screening assay and developed a series of OBA derivatives (molecules 12-15) [23]. OBA was revealed to be a competitive, reversible, active-site directed PTP1B inhibitor [23]. Novo Nordisk attempted further optimization of the OBA scaffold by substituting various aromatic and heteroaromatic systems. Among this series compound 16 and 17 emerged as promising targets [24].
Due to the highly polar nature of pTyr mimetics a common concern associated with them was low cell membrane permeability. As a result, several attempts were made to develop inhibitors with a great cell membrane permeability profile. Li et al. identified a series of lipid like salicylic acid-based derivatives as potent and membrane-permeable PTP1B inhibitors (18-20, Figure 7). They developed bis-anionic amphipathic inhibitors similar to amphiphilic lipids which made them capable of crossing the cell membrane despite the anionic pharmacophore. The acidic part of these compounds bond to the phosphate binding site while the alkyl tails of different lengths were designed to increase hydrophobicity [25].

Liu et al also developed a series of 2-substituted ethenesulfonic acid ester derivatives as potent PTP1B inhibitors possessing sufficient cell membrane permeability (Figure 8). They concluded that an effective, cell permeable PTP1B inhibitor required 4 


\section{Current Research in Diabetes \& Obesity Journal}

essential moieties- (1) a hydrophilic head, (2) an aromatic center, (3) a linker and (4) a hydrophobic tail. 5 series of 2-substituted ethenesulfonic acid ester derivatives were screened to find the optimal structural template containing the abovementioned moieties [26]. The most optimal combination found was with an ethenesulfonic acid ester group as the hydrophilic head, benzene as the aromatic center, a six-carbon linker, and a thiazole ring as the hydrophobic tail (COMP 26c). Various analogs of compound 21 were synthesized with compound 22, containing a pyridyl-2-yl group substitution on the thiazole ring, having excellent PTPT1B inhibitory activity. This study demonstrated that sulfonic acid ester could serve as an effective bioisosteric replacement of the phosphoric acid group.

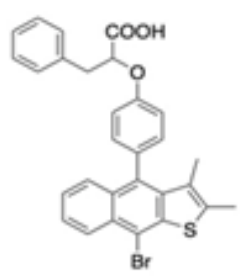

(11)<smiles>O=C(O)C(=O)Nc1ccccc1C(=O)O</smiles><smiles>O=C(O)C(=O)Nc1cc2ccccc2cc1C(=O)O</smiles>

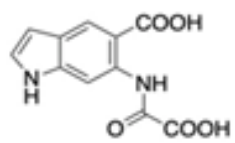

(14)

$\mathrm{IC}_{50}=1.6$ to $29 \mu \mathrm{M}$

$\mathrm{K}_{i}=23 \mu \mathrm{M}$

$\mathrm{K}_{\mathrm{i}}=14 \mu \mathrm{M}$

(depending upon assay conditions)<smiles>O=C(O)C(=O)Nc1ccc(I)cc1C(=O)O</smiles>

(15)

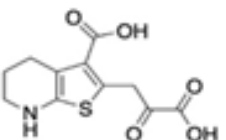

(16)

$\mathrm{K}_{\mathrm{i}}=14 \mu \mathrm{M}$

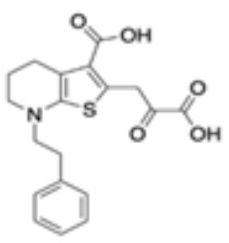

(17)

$\mathrm{IC}_{50}=0.27 \mu \mathrm{M}$

Figure 6: Structures of a mono carboxylic acid benzothiophene pTyr mimetic [19], Ertiprotafib (11) and Novo Nordisk's 2-(oxalylamino)benzoic acid (OBA) derivatives PTP1B inhibitors $(12-17)[23,24]$.

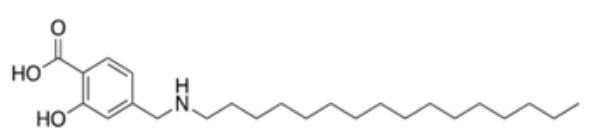

(18)

$\mathrm{IC}_{50}=174 \mu \mathrm{M}$

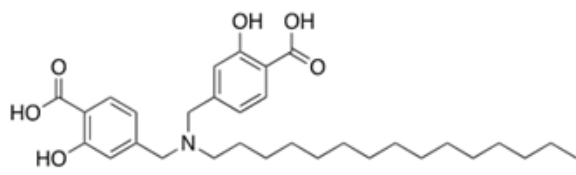

(20)

$\mathrm{IC}_{50}=1.5 \mu \mathrm{M}$

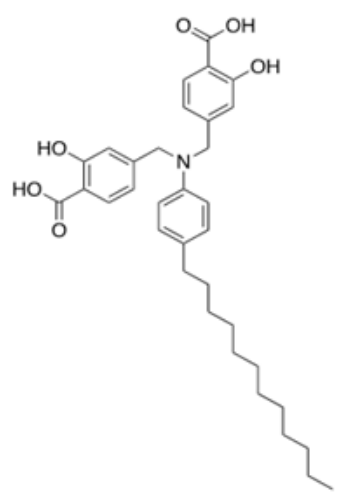

(19)

$\mathrm{IC}_{50}=0.37 \mu \mathrm{M}$

Figure 7: Examples of lipid-like salicylic acid-based derivatives [25].

Apart from the issue of low cell membrane permeability, pTyr mimetic PTP inhibitors also face an issue of selectivity for PTP1B over other PTPs, especially T-cell protein tyrosine phosphatase (TCPTP). TCPTP is a type of phosphatase that is abundantly expressed in hematopoietic cells and is involved in regulation of T-cell activation [27]. TCPTP and PTP1B share a high 
homology in their catalytic domain $(72 \%$ sequence identity and $86 \%$ similarity) 1 with $100 \%$ sequence identity in the phosphate binding region. Hence, inhibitors that bind exclusively to the catalytic pocket or the A-site fail to achieve selectivity [27].

\section{Selective PTP1B Inhibitors}

A number of molecules were developed to target binding to sites other than just the A-site in order to achieve PTP1B selectivity over TCPTP. The Liu group identified inhibitors that are 24-fold more selective to PTP1B over TCPTP (Figure 9) [28]. The salicylate carboxylate group was found to be responsible for its selectivity and is involved in hydrogen bonding interactions with the B-site Arg 24, Arg 254, and Gly 262. Removal of the salicylate carboxylate group resulted in decreased selectivity [28]. Examples of other molecules with B-site interactions that show PTP1B selectivity over TCPTP include isothiazolidinone and salicylic acid containing compound 23, 24 [29], compound 25, and 26 [30]. These identify Arg 24 and 254 as important target residues in providing selectivity.

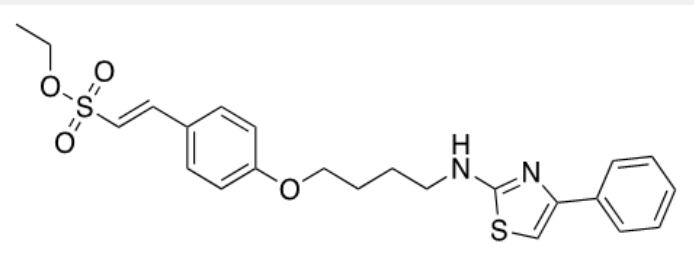

(21)

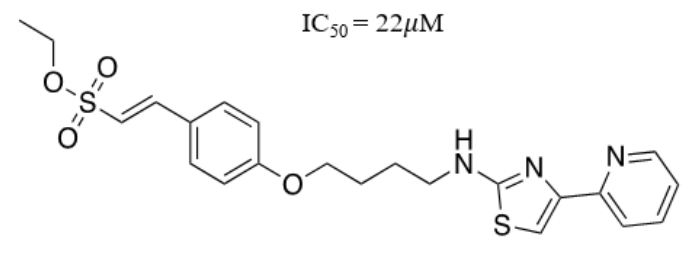

(22)

$$
\mathrm{IC}_{50}=1.5 \mu \mathrm{M}
$$

Figure 8: Examples of substituted ethenesulfonic acid ester derivatives [26].<smiles>COC(=O)c1c(O)cccc1OC/C=C/c1ccc(F)c(-c2cc(C(=O)O)no2)c1</smiles>

(23)

PTPIB IC $\mathrm{C}_{50}=6.9 \mu \mathrm{M}$

TCPTP IC $_{50}=164 \mu \mathrm{M}$

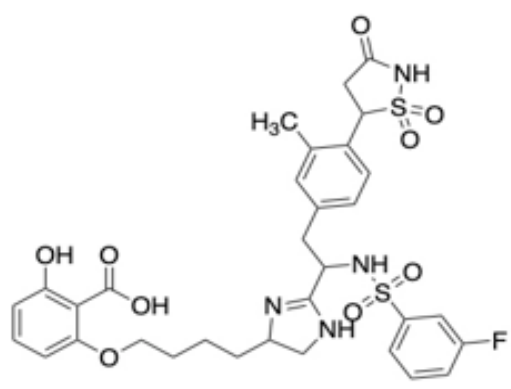

(25)

PTP1B IC so $_{50}=0.022 \mu \mathrm{M}$

TCPTP IC $_{50}=0.046 \mu \mathrm{M}$<smiles>COC(=O)c1c(O)cccc1OC/C=C/c1cccc(-c2onc(C(=O)O)c2CO)c1</smiles>

(24)

PTPIB IC $\mathrm{C}_{50}=0.9 \mu \mathrm{M}$

TCPTP IC $_{50}=19.2 \mu \mathrm{M}$<smiles>CC(=O)NC(Cc1ccc(N(C(=O)C(=O)O)c2ccccc2C(=O)O)c2ccccc12)C(=O)NCCCCCOc1cccc(O)c1C(=O)O</smiles>

(26)

PTP1B IC $\mathrm{C}_{50}-0.022 \mu \mathrm{M}$

TCPTP IC $_{50}=0.049 \mu \mathrm{M}$

Figure 9: Examples of salicylic acid based selective PTP1B inhibitors [28-30]. 
Other important B-site residues aiding selectivity include Phe 52, Cys 32, and Met 258. Abbott laboratories identified compound 27 as a potent PTP1B inhibitor with 4-fold selectivity over TCPTP (Figure 10) [31]. It was observed that its salicylate group, in addition to its interactions with Arg 24 and 254, was also accessible to Phe 52, [31] a residue which is not conserved between PTP1B and TCPTP. The 4-chloro substituted variant of compound 27 was 6-fold more selective due to its additional hydrophobic interaction with Phe 52. Therefore, it could be hypothesized that interactions with Phe 52 could further enhance selectivity. Although forming interactions with other B-site residues such as Met 258 and Gly 259 served as a promising strategy to further increase selectivity, the results for these interactions remain inconsistent. Compound 28 developed by Wan group, where the sulfonyl oxygens formed water-mediated H-bond interactions with Arg 24 and 254 and the benzyl group forming hydrophobic interactions with Phe52 did not show selectivity over TCPTP [32]. As a result, there were several attempts to better analyze the effects of each of these interactions on selectivity.

It was observed that hydrophobic substituents of appropriate size and length interact with the non-polar B-site residues providing selectivity. Lakshminarayana et al. developed a series of novel, nonphosphoric acid containing PTP1B inhibitors (compounds 29, 30 and 31) out of which 31 was nearly 20fold more selective to PTP1B than TCPTP (Figure 11). It was hypothesized that the molecule's hydrophobic side chain fully occupied the B-site with the pyrrolidino group being key in providing selectivity [33]. Compound 32 developed by Li group is another example of a selective compound that forms hydrophobic interactions with the B-site of PTP1B [27]. It had 10 times greater selectivity for PTP1B compared to TCPTP.<smiles>C=CCOC(=O)N[C@H](Cc1ccc(C(C(=O)C(=O)O)c2ccccc2C(=O)O)cc1)C(=O)NCCCCOc1cccc(O)c1C(=O)O</smiles>

(27)

PTPIB IC ${ }_{50}=0.120 \mu \mathrm{M}$

$\mathrm{TCPTP}_{\mathrm{IC}}=0.470 \mu \mathrm{M}$

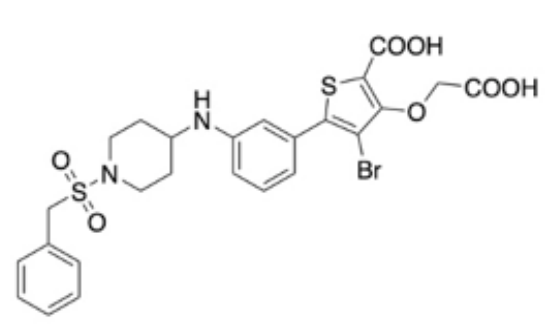

(28)

PTPIB IC ${ }_{50}=0.004 \mu \mathrm{M}$

TCPTP IC $_{50}=0.005 \mu \mathrm{M}$

Figure 10: Compound 27 developed by Abbott laboratories [31] and Compound 28 developed by Wan group [32].
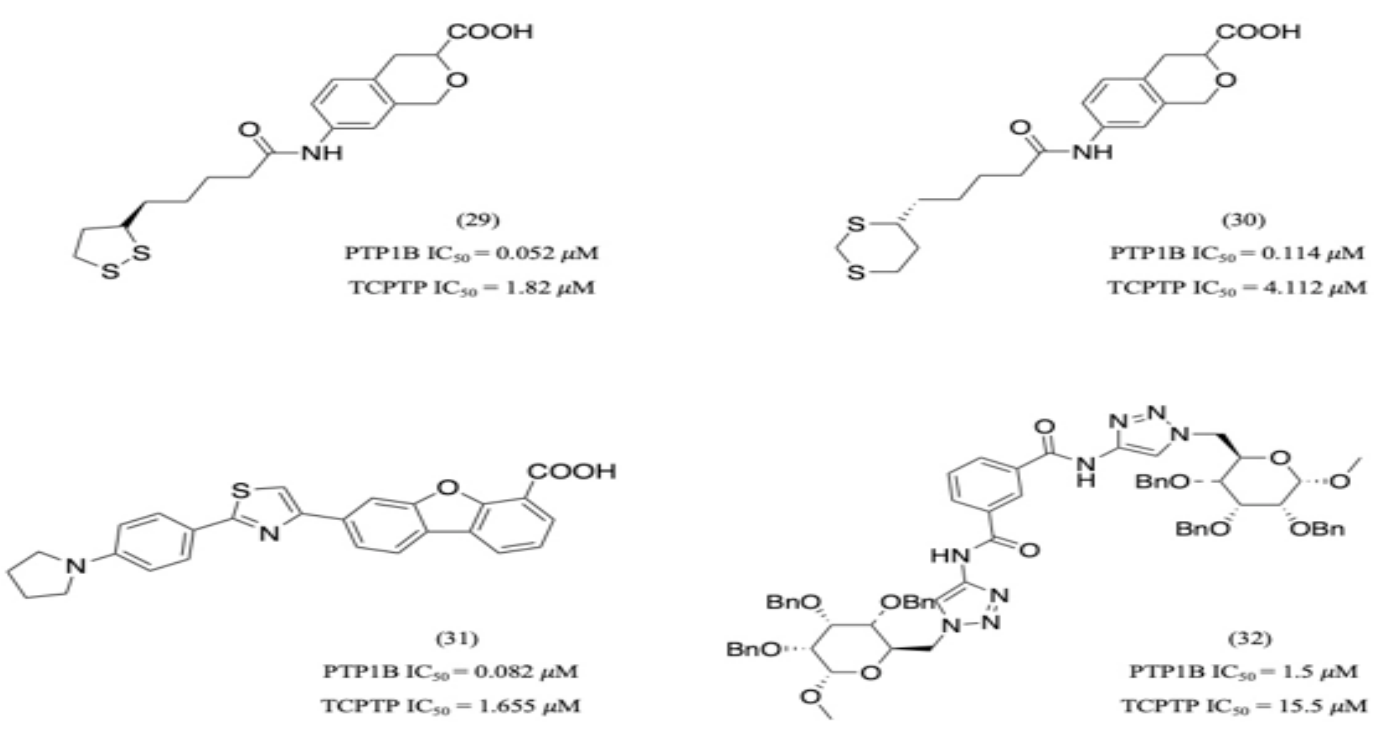

Figure 11: Compound 29, 30 and 31 developed by Lakshminarayana et al. [33] and compound 32 developed by Li group [27]. 
Additionally, Cys 32 in site B was also identified as a critical residue to provide selectivity. Cys 32 is not conserved on PTP1B and TCPTP [27]. Molecular modelling studies of bidentate $\alpha$-ketoacid-based inhibitors identified the importance of this residue [34]. The most active compound of the series 33 was found to interact with Cys 32 (Figure 12). Within the C-site, Arg
47, Asp 48, and Lys 41 were identified as crucial targets to obtain selectivity [35]. However, compounds developed early on such as compound 34 by the Wan group [35], compound 35 by the Sun group [36] and compound 36 by the Liu group [37] did interact with these residues but failed to provide significant selectivity (Figure 13).

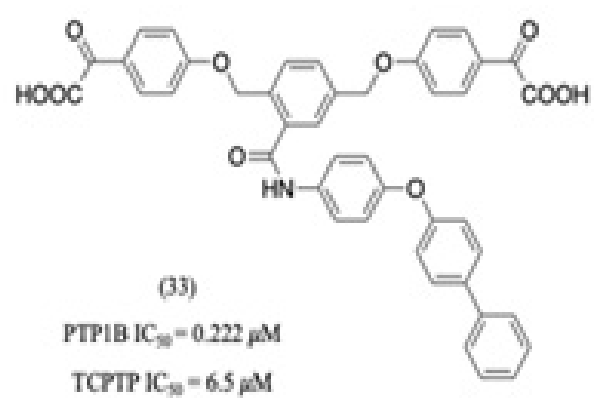

(A)

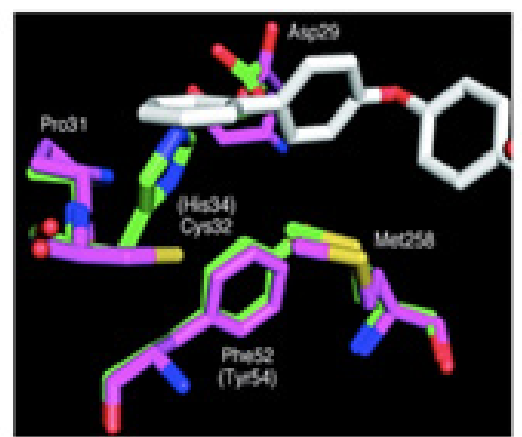

(B)

Figure 12: Compound $33(\mathrm{~A})$ and its molecular modelling studies (B) depicting interaction with Cys 32 [Image taken from reference 34].

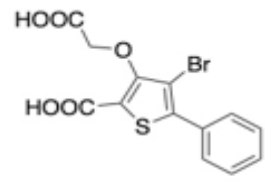

(34)

PTP1B IC ${ }_{50}=0.30 \mu \mathrm{M}$

TCPTP IC ${ }_{50}=0.32 \mu \mathrm{M}$

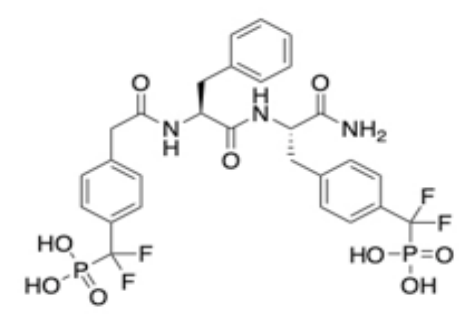

(35)

PTP1B IC s0 $_{50}=0.004 \mu \mathrm{M}$

TCPTP IC SO $_{S_{0}}=0.020 \mu \mathrm{M}$

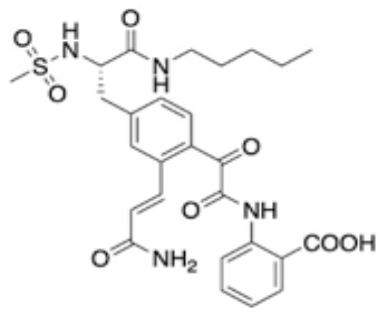

(36)

PTP1B IC s0 $=0.17 \mu \mathrm{M}$

TCPTP IC $_{s_{0}}=0.16 \mu \mathrm{M}$

Figure 13: Compound 34 developed by the Wan group [35], compound 35 by the Sun group [36] and compound 36 by the Liu group [36] interacting with the C-site, Arg 47, Asp 48, and Lys 41.

A series of triaryl-sulfonamides was reported by Patel group with their most potent being compound 37 displayed 96-fold selectivity for PTP1B over TCPTP [38]. Docking studies revealed that the molecule's 2-phenoxyacetic acid moiety formed hydrogen bonds with Asp 48 while the thiadiazoyl moiety interacts with Arg 24 and 254 of the B-site. Li group developed a series of thiazoylbased PTP1B inhibitors which contained a glucosyl as a chiral linker. Compound 38 showed 6-fold selectivity for PTP1B over TCPTP (Figure 14). It was revealed to interact with Asp 47, Asp 48, and Arg 24 [39].

Critical residues providing PTP1B selectivity within the D-site include Tyr 46, Lys 116, Lys 119and Lys 12027. Since the D-site is located fairly close to the catalytic site, inhibitors targeting the D-site are typically less bulky compared to those targeting other sites. Compound 39 a 2-(oxalyl amino) benzoic acid derivative developed by Andersen group is an example of molecule targeting the D-site which displayed 26 times more selectivity towards PTP1B than TCPTP (Figure 14) [40]. Additionally, PTP1B is the only tyrosine specific phosphatase that has a Leu residue at the $119^{\text {th }}$ position [27]. Asante-Appiach et al. identified compound 40 as a PTP1B inhibitor with 2- fold selectivity over TCPTP [41] (Figure 15). X-ray crystal structure analysed that the benzyl ester group interacts with Tyr 46, Ser118 and Leu 119. A mutant study was carried out to confirm the role of Leu 119 in 
providing selectivity by substituting it with Val. This substitution had diminished selectivity. Further structural modifications to
Compound 40 yielded Compound 41 which was found to be 10 fold more selective towards PTP1B over TCPTP [41].<smiles>O=C(O)COc1ccc(S(=O)(=O)N(Cc2ccc(-c3cnns3)cc2)Cc2ccc3cc(F)c(C(F)(F)[OH+]O[O-])cc3c2)cc1</smiles>

(37)

PTP1B IC $50=0.009 \mu \mathrm{M}$

TCPTP IC $50=0.87 \mu \mathrm{M}$<smiles>COC(=O)C(Cc1ccc(O)cc1)n1cc(CO[C@H]2C(Cc3ccccc3)C[C@H](OCc3ccccc3)C(OCc3ccccc3)[C@H]2OCc2cn(C(Cc3ccccc3)C(C)=O)nn2)nn1</smiles>

(38)

PTP1B IC ${ }_{90}=9.6 \mu \mathrm{M}$

TCPTP IC $\mathrm{C}_{50}=59.6 \mu \mathrm{M}$

Figure 14: Compound 37 reported by Patel group [38] and compound 38 developed by the Li group [39].

$\mathrm{Du}$ et al discovered ADC type inhibitors which interacted with the A, D, and C site of PTP1B. Their drug design started with compound 42 containing a DFMP scaffold which interacted with Lys 41 and Arg 47 of the C-site (Figure 16) [13]. Based on this, fragment 2 was selected for further optimization. Since the DFMP scaffold is known to have poor cell permeability, it was replaced with a different hydrogen bond acceptor i.e. a methyl sufonly fragment (fragment 3). Docking studies indicated that the oxygens of the 2 sulfonly groups hydrogen bond to Ser 216, Ile 219, Gly220, Arg 221. Binding to Asp 48 of the A-site was known to enhance selectivity. Hence, a urea group was introduced to interact with this residue (fragment 4). Furthermore, docking studies suggested that substitution on the $2^{\text {nd }}$ and $5^{\text {th }}$ position of the phenyl ring of fragment 4 would provide a means to interact with the hydrophobic D-site residues (compound 43). The additional
2 ethoxy groups introduced in compound 43 interacted with Val 49, Phe 52, Ile219, and Met 258 of the D-site. Further analogs of compound 43 were synthesized and Compound 43, with an IC50 of 203 nM showed 120-fold PTP1B selectivity over TCPTP.13

Yang et al developed a series of ethene sulfonic acid ester (the non-ionic bioisostere of phosphoric acid) containing compounds [42]. Among various analogs synthesized Y-shaped bis-arylethenesulfonic acid ester derivatives were the most potent and membrane permeable (Figure 17). Attempts were made to develop more selective compound with the most potent being compound 44 IC $_{50}$ of $140,1290 \mathrm{nM}$ for PTP1B and TCPTP respectively). Docking studies revealed that in addition to the active site, the compound also interacted with the $\mathrm{C}$ and $\mathrm{E}$ site [42], which could explain its selectivity.

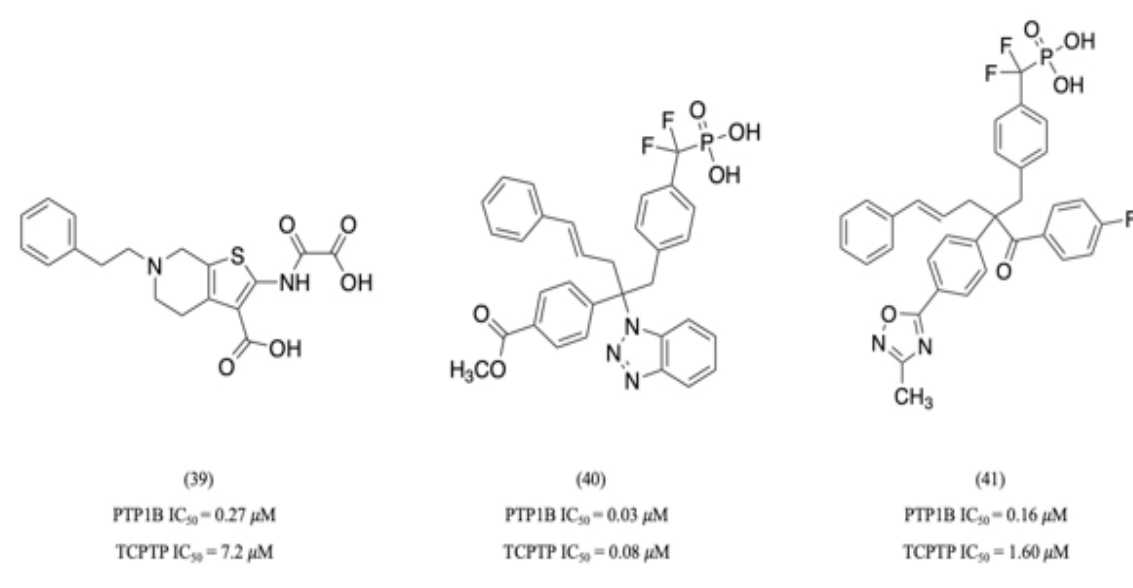

Figure 15: Compound 39 developed by Andersen group [40] and compound 40 and 41 developed by Asante-Appiach et al. [41]. 

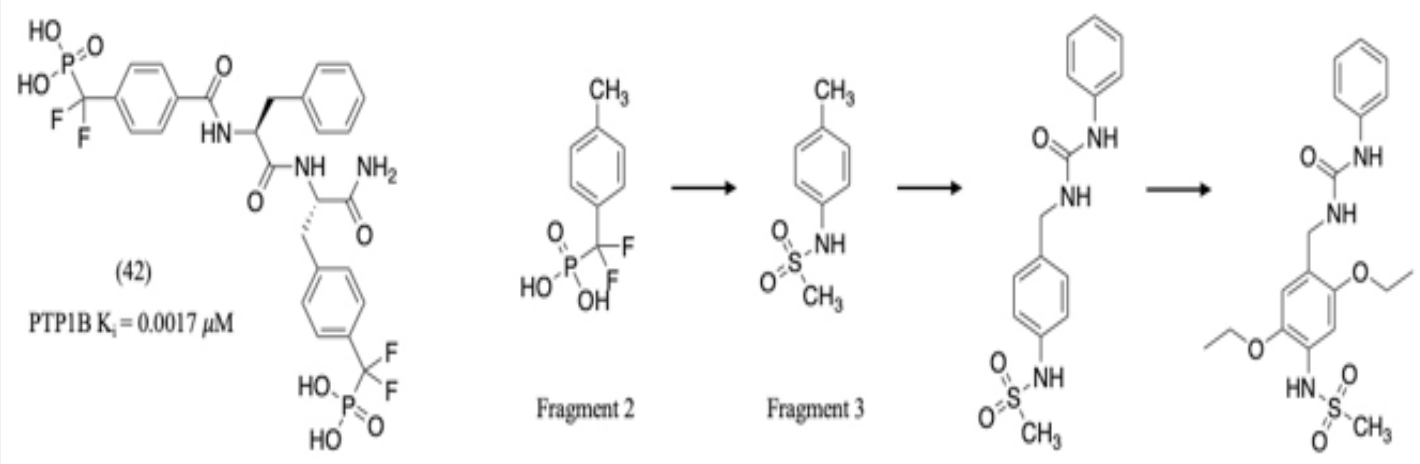

Fragment 4

(43)

PTPIB IC s0 $_{0}=0.20 \mu \mathrm{M}$

Figure 16: Drug design of ADC type inhibitors by Du et al. [13].

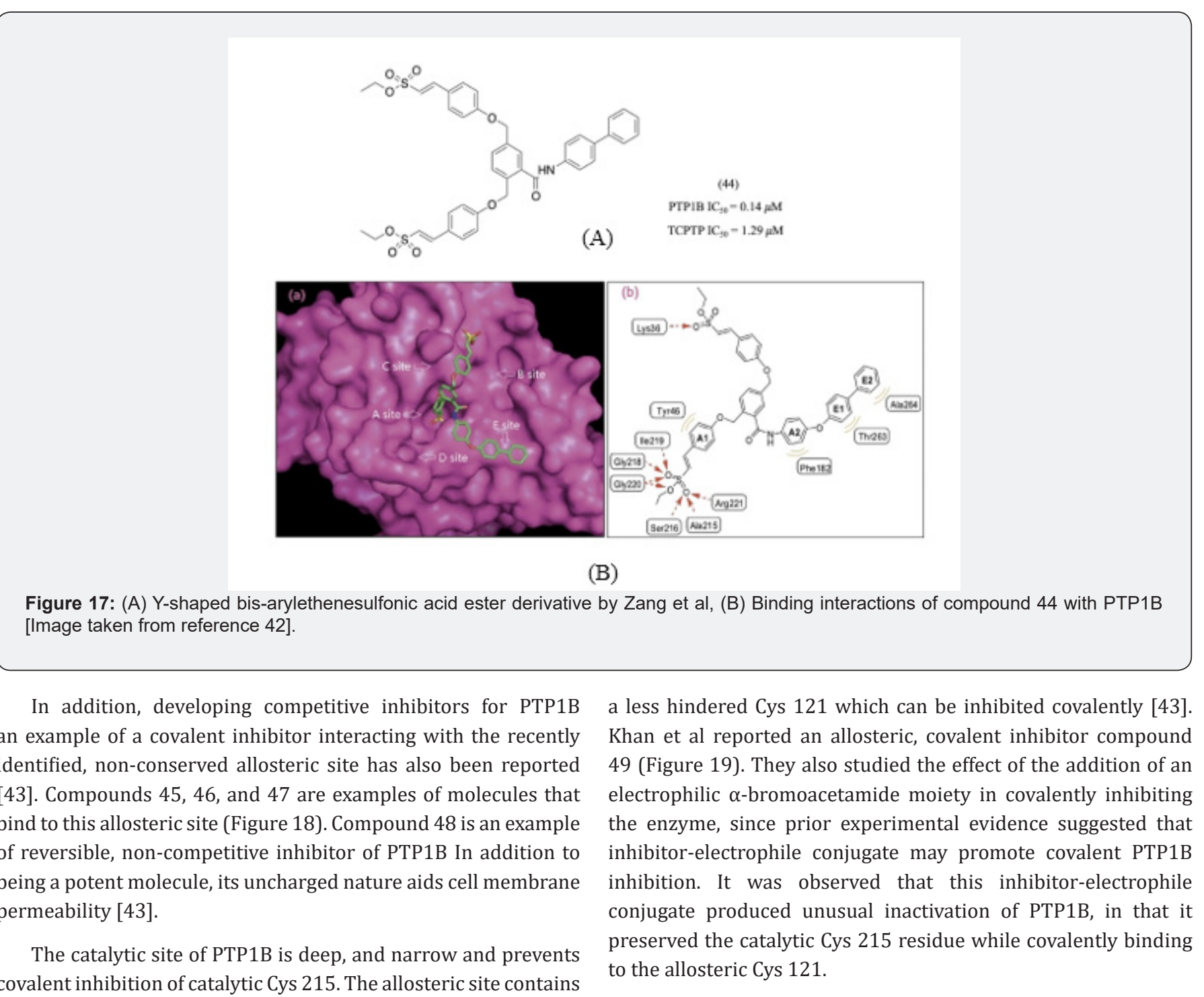
covalent inhibition of catalytic Cys 215. The allosteric site contains 


\section{Current Research in Diabetes \& Obesity Journal}

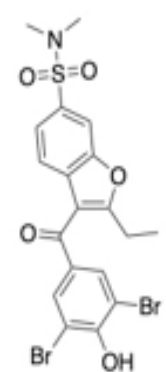

(45)

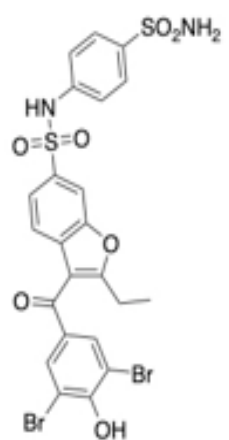

(46)

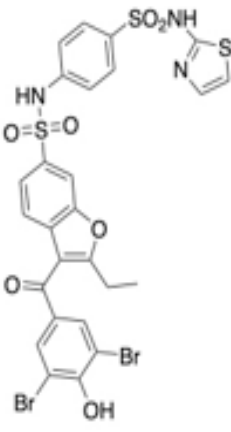

(47)

PTPIB IC . $_{90}=8 \mu \mathrm{M}$

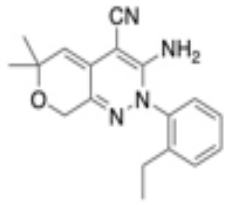

(48)

PTPIB IC $50=350 \mu \mathrm{M}$

PTP1B IC ${ }_{50}=22 \mu \mathrm{M}$

Figure 18: Examples of compounds that bind to this allosteric site on PTP1B [43]

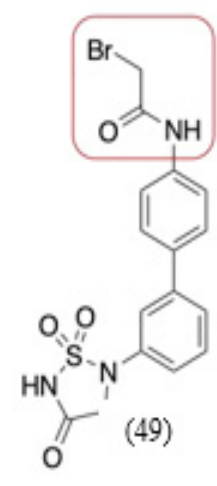

(A)

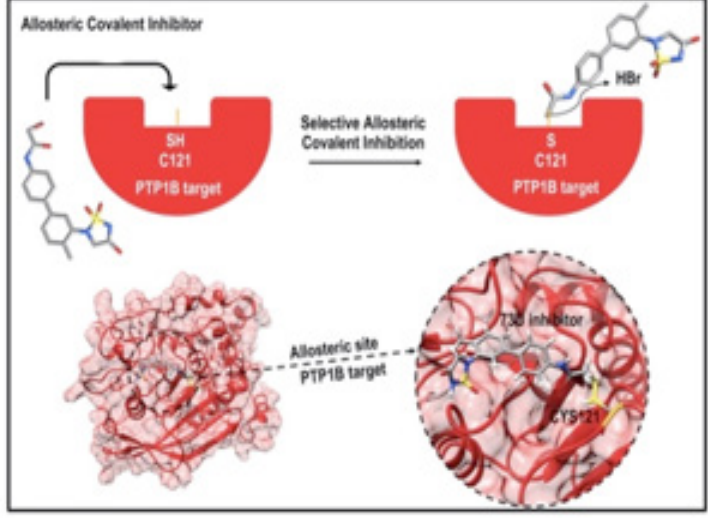

(B)

Figure 19: (A) Allosteric, covalent inhibitor developed by Khan et al with the $\square$-bromoacetamide moiety highlighted. (B) Interaction of compound 49 with allosteric site of PTP1B [Image taken from reference 43].

\section{Conclusion}

Early active site directed PTP1B inhibitors faced the limitation of poor cell membrane permeability. This issue was tackled by developing either un-charged or amphoteric inhibitors. However, these inhibitors mainly interacted with the active site of the enzyme and hence failed to provide selectivity over other PTPs, especially T-cell protein tyrosine phosphatase (TCPTP). PTP1B and TCPTP share high homology in their catalytic domain $(72 \%$ sequence identity and $86 \%$ similarity) 1 and $100 \%$ sequence identity in the phosphate binding region. Therefore, in order to develop more selective inhibitors, molecules were designed to bind to sites other than just the PTP1B active site. This approach identified certain residues as 'critical'. Binding interactions with these residues helped to provide PTP1B selectivity over TCPTP. Along with several strategies to inhibit PTP1B competitively, an example of a covalent inhibition has also been reported. A secondary allosteric site on PTP1B has been recently identified which contains Cys 121 which is more accessible compared to the active site Cys215. Khan et al reported an allosteric, covalent inhibitor binding to Cys 121 . Hence, providing an alternative residue to covalently target other than the highly hindered catalytic site Cys 215. Hence, constant developments and new strategies continue to emerge towards developing effective and selective PTP1B inhibitors and this continues to serve as an active area of research.

\section{Acknowledgement}

The authors wish to thank the School of Pharmacy at the Massachusetts College of Pharmacy and Health Sciences University for financial support of this project. 


\section{Current Research in Diabetes \& Obesity Journal}

\section{References}

1. Tiganis T (2013) PTP1B and TCPTP - Nonredundant Phosphatases in Insulin Signaling and Glucose Homeostasis: Regulation of Insulin Signaling by PTP1B and TCPTP. FEBS J 280 (2): 445-458.

2. Kerru N, Singh-Pillay A, Awolade P, Singh P (2018) Current AntiDiabetic Agents and Their Molecular Targets: A Review. Eur J Med Chem 152: 436-488.

3. (2019) Diabetes Quick Facts.

4. Verma M, Gupta SJ, Chaudhary A, Garg VK (2017) Protein Tyrosine Phosphatase 1B Inhibitors as Antidiabetic Agents - A Brief Review. Bioorganic Chem 70: 267-283.

5. Garcia-Barrado MJ, Jonas JC, Gilon P, Henquin JC (1996) Sulphonylureas Do Not Increase Insulin Secretion by a Mechanism Other than a Rise in Cytoplasmic Ca2+ in Pancreatic B-Cells. Eur J Pharmacol 298(3): 279286.

6. Malinowski JM, Bolesta S (2000) Rosiglitazone in the treatment of type 2 Diabetes Mellitus: A Critical Review. 22(10): 1151-1168.

7. Fukaya N, Mochizuki K, Tanaka Y, Kumazawa T, Jiuxin Z, et al. (2009) The $\alpha$-Glucosidase Inhibitor Miglitol Delays the Development of Diabetes and Dysfunctional Insulin Secretion in Pancreatic $\beta$-Cells in OLETF Rats. Eur J Pharmacol 624 (1-3): 51-57.

8. Knudsen LB (2004) Glucagon-like Peptide-1: The Basis of a New Class of Treatment for Type 2 Diabetes. J Med Chem 47(17): 4128-4134.

9. (2020) Gestational diabetes - Symptoms and causes - Mayo Clinic

10. Kim SJ, Ryu SE (2012) Structure and Catalytic Mechanism of Human Protein Tyrosine Phosphatome. BMB Rep 45(12): 693-699.

11. Sarath Kumar B, Lakshmi, BS (2019) In Silico Investigations on the Binding Efficacy and Allosteric Mechanism of Six Different Natural Product Compounds towards PTP1B Inhibition through Docking and Molecular Dynamics Simulations. J Mol Model 25(9): 272.

12. Ala PJ, Gonneville L, Hillman M, Becker-Pasha M, Yue EW, et al. (2006) Structural Insights into the Design of Nonpeptidic IsothiazolidinoneContaining Inhibitors of Protein-Tyrosine Phosphatase 1B. J Biol Chem 281(49): 38013-38021.

13. Du Y, Zhang Y, Ling H, Li Q, Shen J (2018) Discovery of Novel High Potent and Cellular Active ADC Type PTP1B Inhibitors with Selectivity over TC-PTP via Modification Interacting with C Site. Eur J Med Chem 144: $692-700$

14. Sharma B, Xie L, Yang F, Wang W, Zhou Q, et al. (2020) Recent Advance on PTP1B Inhibitors and Their Biomedical Applications. Eur J Med Chem 199: 112376

15. Nam HJ, Poy F, Krueger NX, Saito H, Frederick CA (1999) Crystal Structure of the Tandem Phosphatase Domains of RPTP LAR. 97(4): 449-457.

16. Brandão TAS, Hengge AC, Johnson SJ (2010) Insights into the Reaction of Protein-Tyrosine Phosphatase 1B: Crystal Structures for Transition State Analogs of Both Catalytic Steps. J Biol Chem 285(21): 1587415883.

17. The Insulin Receptor and Its Signal Transduction Network - Endotext.

18. Koren S, Fantus IG (2007) Inhibition of the Protein Tyrosine Phosphatase PTP1B: Potential Therapy for Obesity, Insulin Resistance and Type-2 Diabetes Mellitus. Best Pract Res Clin Endocrinol Metab 21(4): 621-640.

19. Elsa Irving, Andrew Stoker (2017) Vanadium Compounds as PTP Inhibitors. Molecules 22(12): 2269.
20. Ye B, Akamatsu M, Shoelson SE, Wolf G, Giorgetti-Peraldi S, et al (1995) L-O-(2-Malonyl) Tyrosine: A New Phosphotyrosyl Mimetic for the Preparation of Src Homology 2 Domain Inhibitory Peptides. J Med Chem 38(21): 4270-4275.

21. Burke TR, Ye B, Akamatsu M, Ford H, Yan X, et al. (1996) P. P. 4'- O -[2-(2-Fluoromalonyl)]- L -Tyrosine: A Phosphotyrosyl Mimic for the Preparation of Signal Transduction Inhibitory Peptides 1. J Med Chem 39(5): 1021-1027.

22. Shrestha S, Bhattarai BR, Cho H, Choi JK, Cho H (2007) PTP1B Inhibitor Ertiprotafib Is Also a Potent Inhibitor of IкB Kinase $\beta$ (IKK- $\beta$ ). Bioorg Med Chem Lett 17(10): 2728-2730.

23. Andersen HS, Iversen LF, Jeppesen CB, Branner S, Norris K, et al. (2000) 2-(Oxalylamino)-Benzoic Acid Is a General, Competitive Inhibitor of Protein-Tyrosine Phosphatases. J. Biol. Chem 275(10): 7101-7108.

24. Lund IK, Andersen HS, Iversen LF, Olsen OH, Møller KB, et al. (2004) Structure-Based Design of Selective and Potent Inhibitors of ProteinTyrosine Phosphatase $\beta$. J Biol Chem 279(23): 24226-24235.

25. Li L, Tavallaie MS, Xie F, Xia Y, Liang Y, et al. (2019) Identification of Lipid-like Salicylic Acid-Based Derivatives as Potent and MembranePermeable PTP1B Inhibitors. Bioorganic Chem 93: 103296.

26. Liu J, Jiang F, Jin Y, Zhang Y, Liu J, et al. (2012) Design, Synthesis, and Evaluation of 2-Substituted Ethenesulfonic Acid Ester Derivatives as Protein Tyrosine Phosphatase 1B Inhibitors. Eur J Med Chem 57: 1020.

27. Li X, Wang L, Shi D (2016) The Design Strategy of Selective PTP1B Inhibitors over TCPTP. Bioorg Med Chem 24(16): 3343-3352.

28. Liu G, Xin Z, Pei Z, Hajduk PJ, Abad-Zapatero C, et al. (2003) Fragment Screening and Assembly: A Highly Efficient Approach to a Selective and Cell Active Protein Tyrosine Phosphatase 1B Inhibitor. J Med Chem 46(20): 4232-4235.

29. Douty B, Wayland B, Ala PJ, Bower MJ, Pruitt J, et al. (2008) Isothiazolidinone Inhibitors of PTP1B Containing Imidazoles and Imidazolines. Bioorg Med Chem Lett 18(1): 66-71.

30. Szczepankiewicz BG, Liu G, Hajduk PJ, Abad-Zapatero C, Pei Z, et al. (2003) Discovery of a Potent, Selective Protein Tyrosine Phosphatase 1B Inhibitor Using a Linked-Fragment Strategy. J Am Chem Soc 125(14): 4087-4096.

31. Ye D, Zhang Y, Wang F, Zheng M, Zhang X, et al. (2010) Novel Thiophene Derivatives as PTP1B Inhibitors with Selectivity and Cellular Activity. Bioorg Med Chem 18(5): 1773-1782.

32. Wilson DP, Wan ZK, Xu WX, Kirincich SJ, Follows BC, et al. (2007) Structure-Based Optimization of Protein Tyrosine Phosphatase 1B Inhibitors: From the Active Site to the Second Phosphotyrosine Binding Site. J Med Chem 50(19): 4681-4698.

33. Lakshminarayana N, Rajendra Prasad Y, Gharat L, Thomas A, Ravikumar P, et al. (2009) Synthesis and Evaluation of Some Novel Isochroman Carboxylic Acid Derivatives as Potential Anti-Diabetic Agents. Eur J Med Chem 44(8): 3147-3157.

34. Comeau AB, Critton DA, Page R, Seto CT (2010) A Focused Library of Protein Tyrosine Phosphatase Inhibitors. J Med Chem 53(18): 67686772.

35. Wan ZK, Lee J, Xu W, Erbe DV, Joseph-McCarthy D, et al. (2006) Monocyclic Thiophenes as Protein Tyrosine Phosphatase 1B Inhibitors: Capturing Interactions with Asp48. Bioorg Med Chem Lett 16(18): 4941-4945.

36. Sun JP, Fedorov AA, Lee SY, Guo XL, Shen K, et al. (2003) Crystal Structure of PTP1B Complexed with a Potent and Selective Bidentate Inhibitor. J Biol Chem 278(14): 12406-12414. 


\section{Current Research in Diabetes \& Obesity Journal}

37. Liu G, Szczepankiewicz BG, Pei Z, Janowick DA, Xin Z, et al (2003) Discovery and Structure-Activity Relationship of Oxalylarylaminobenzoic Acids as Inhibitors of Protein Tyrosine Phosphatase 1B. J Med Chem 46(11): 2093-2103.

38. Patel D, Jain M, Shah SR, Bahekar R, Jadav P, et al. (2012) Discovery of Potent, Selective and Orally Bioavailable Triaryl-Sulfonamide Based PTP1B Inhibitors. Bioorg Med Chem Lett 22(2): 1111-1117.

39. He XP, Li C, Jin XP, Song Z, Zhang HL, et al. (2011) Microwave-Assisted Construction of Triazole-Linked Amino Acid-Glucoside Conjugates as Novel PTP1B Inhibitors. New J Chem 35(3): 622.

40. Andersen HS, Olsen OH, Iversen LF, Sørensen ALP, Mortensen SB, et al. (2002) Discovery and SAR of a Novel Selective and Orally Bioavailable Nonpeptide Classical Competitive Inhibitor Class of Protein-Tyrosine Phosphatase 1B. J Med Chem 45(20): 4443-4459.
41. Asante-Appiah E, Patel S, Desponts C, Taylor JM, Lau C, et al. (2006) Conformation-Assisted Inhibition of Protein-Tyrosine Phosphatase-1B Elicits Inhibitor Selectivity over T-Cell Protein-Tyrosine Phosphatase. J Biol Chem 281(12): 8010-8015.

42. Yang F, Xie F, Zhang Y, Xia Y, Liu W, et al. (2017) Y-Shaped BisArylethenesulfonic Acid Esters: Potential Potent and Membrane Permeable Protein Tyrosine Phosphatase 1B Inhibitors. Bioorg Med Chem Lett 27(10): 2166-2170.

43. Khan S, Bjij I, Soliman MES (2019) Selective Covalent Inhibition of "Allosteric Cys121" Distort the Binding of PTP1B Enzyme: A Novel Therapeutic Approach for Cancer Treatment. Cell Biochem Biophys $77(3): 203-211$

Your next submission with Juniper Publishers
will reach you the below assets
- Quality Editorial service
- Swift Peer Review
- Reprints availability
- E-prints Service
- Manuscript Podcast for convenient understanding
- Global attainment for your research
- Manuscript accessibility in different formats
- Undf, E-pub, Full Text, Audio)
Track the below URL for one-step submission
https://juniperpublishers.com/online-submission.php

